02

\title{
Решение обратной задачи для сложного вибронного аналога резонанса Ферми на основе плоских вращений Якоби
}

\author{
(C) В.А. Кузьмицкий \\ Университет гражданской защиты МЧС Республики Беларусь, \\ 220118 Минск, Беларусь \\ e-mail: llum07@rambler.ru
}

Поступила в редакцию 10.01.2020 г.

В окончательной редакции 25.06.2020 г.

Принята к публикации 28.07.2020 г.

На основе алгебраических методов найдено точное решение обратной задачи для сложного вибронного аналога резонанса Ферми, т. е. определения из спектральных данных для наблюдаемого конгломерата линий (энергий $E_{k}$ и интенсивностей переходов $I_{k}, k=1,2, \ldots, n ; n>2$ ) энергий „темных“ состояний $A_{m}$ и матричных элементов их связи $B_{m}$ со „светлым“ состоянием. В первой части алгоритма с помощью плоских вращений Якоби находится ортогональная матрица преобразования подобия $X$, элементы первой строки которой подчинены условию $\left(X_{1 k}\right)^{2}=I_{k}$ в соответствии с тем, что только одно невозмущенное состояние является „светлым“. Во второй части величины $A_{m}$ и $B_{m}$ получаются из решения задачи на собственные значения для блока „темных“ состояний матрицы $X \operatorname{diag}\left(\left\{E_{k}\right\}\right) X^{-1}$.

Ключевые слова: электронно-колебательное взаимодействие, сложный резонанс Ферми, обратная задача, плоские вращения Якоби.

DOI: $10.21883 /$ OS.2020.11.50163.3-20

\section{Введение}

Электронно-колебательное взаимодействие в условиях резонанса с участием возбужденных электронных состояний $S_{2}$ и $S_{1}-$ вибронный аналог сложного резонанса Ферми (термин Герцберга [1]) - экспериментально тщательно исследовано Весселем и Мак Клюром [2]. ${ }^{1}$ Ими обнаружено, что в низкотемпературных спектрах поглощения молекул нафталина и его дейтерированного производного, внедренных в качестве примесного центра в кристаллы дурола и ксилола, вместо одной линии, отвечающей 0-0-переходу во второе синглетное состояние $S_{0} \rightarrow S_{2}$, возникает запутанный „конгломерат“ из нерегулярно расположенных узких линий с числом компонент не менее 50. Перераспределение 0-0-перехода $S_{0} \rightarrow S_{2}$ по большому числу линий наблюдается также в высокоразрешенных низкотемпературных спектрах поглощения (возбуждения люминесценции) ряда ароматических и гетероароматических соединений: феназина и акридина [3], 4Н-пиран-4-тиона [4], пирена [5,6], бенз[ghi]перелена [7,8], террилена [9], индола [10], молекул класса порфиринов [11-14]. Аналогичным образом с проявлением вибронного взаимодействия с участием нижних триплетных состояний $T_{1}$ и $T_{2}$ связаны особенности наблюдаемых при низких температурах спектров возбуждения фосфоресценции примесных центров бензальдегида в кристаллах ацетофенона [15], метилбензальдегида в кристаллах дурола [16], их дейтерированных производных, кристалла ацетофенона [17]. Спин-

\footnotetext{
1 Результаты работы [2] 1980 г. представлены также в труднодоступной диссертации Весселя 1971 г.
}

орбитальной и вибронной связью первого возбужденного синглетного состояния $S_{1}$ с близкими по энергии колебательно возбужденными состояниями триплетного состояния объяснены результаты измерений вращательно разрешенных спектров возбуждения флуоресценции пиразина [18,19], метил-производных пиримидина [20].

Наибольший интерес в приведенных примерах представляют сведения о электронно-колебательных уровнях и матричных элементах вибронных резонансных (квазирезонансных) взаимодействий. Попытки расчета сложных спектров, возникающих в результате вибронного взаимодействия, на основе методов квантовой химии предпринимались в работах $[5,7,17]$. Однако решение этой задачи сильно осложняется требованием высокой точности расчета электронных и колебательных состояний, необходимой для выполнения условия резонанса. Кроме того, экспериментальные данные свидетельствуют о существенном влиянии окружения молекулы на наблюдаемое распределение интенсивности линий, так что интервал энергии между „взаимодействующими“ электронными уровнями является своеобразным параметром модуляции резонанса $[12,13,15]$. Поэтому может быть сформулирована обратная задача, которая предусматривает определение из экспериментальных данных матричных элементов резонансных вибронных взаимодействий. Следует отметить, что аналогичная обратная задача актуальна для описания ряда других явлений, характеризуемых близостью уровней различной физической природы, например внутримолекулярного перераспределения колебательной энергии [21] (сложный резонанс Ферми). 
В настоящей работе показано, что такая обратная задача имеет точное решение, если из экспериментальных данных известны энергии и интенсивности линий наблюдаемого конгломерата при условии, что разрешен только один переход в исходное невозмущенное состояние.

\section{Постановка задачи}

С квантово-механической точки зрения для рассмотрения сложного резонанса Ферми или его вибронного аналога существенно наличие точного или приближенного вырождения состояний $\varphi_{1}$ и $\varphi_{m}(m=2,3, \ldots, n$, $n \geq 3$ ), которые полагают собственными функциями гамильтониана нулевого приближения $\hat{H}^{(0)}$, и „взаимодействия“ между ними за счет возмущения $\hat{H}^{(1)}$. Выделение состояния $\varphi_{1}$ связано с тем, что только один переход из начального (основного) состояния $\varphi_{0} \rightarrow \varphi_{1}$ является разрешенным, а остальные переходы $\varphi_{0} \rightarrow \varphi_{m}$ запрещены. Состояние $\varphi_{1}$ называют „светлым“ (bright), a состояния $\varphi_{m}$ - „темными“ (dark). В рамках линейного вариационного метода волновые функции возмущенных состояний $\Psi_{k}$ представляются как

$$
\Psi_{k}=\sum_{l=1}^{n} C_{l k} \varphi_{l}=C_{1 k} \varphi_{1}+\sum_{m=2}^{n} C_{m k} \varphi_{m} .
$$

Базисные функции $\varphi_{l}$ будем считать действительными, тогда матрица $C$, составленная из коэффициентов $C_{l k}$, будет ортогональной, $C^{-1}=C^{t}$. Здесь и далее индекс $t$ означает транспонирование.

Прямая задача состоит в вычислении матричных элементов

$$
\left\langle\varphi_{k}|\hat{H}| \varphi_{l}\right\rangle=\left\langle\varphi_{k}\left|\hat{H}^{(0)}+\hat{H}^{(1)}\right| \varphi_{l}\right\rangle,
$$

решении секулярной проблемы

$$
H C=C E, E=\operatorname{diag}\left(E_{1}, E_{2}, \ldots, E_{n}\right)
$$

и вычислении интенсивности переходов из основного состояния $\varphi_{0} \rightarrow \Psi_{k}$, пропорциональной квадрату матричного элемента $\left\langle\Psi_{k}|\mathbf{M}| \varphi_{0}\right\rangle, \mathbf{M}$ - оператор дипольного момента молекулы. Так как разрешен только переход $\varphi_{0} \rightarrow \varphi_{1}$, относительная интенсивность переходов в результирующие состояния $\varphi_{0} \rightarrow \Psi_{k}$,

$$
I_{k}=\left|\left\langle\Psi_{k}|\mathbf{M}| \varphi_{0}\right\rangle\right|^{2} /\left|\left\langle\varphi_{1}|\mathbf{M}| \varphi_{0}\right\rangle\right|^{2},
$$

выражается через элементы первой строки матрицы $C$,

$$
I_{k}=\left(C_{1 k}\right)^{2}, \quad k=1,2, \ldots, n .
$$

Из (1), (2) следует, что выбор базисных функций „темных“ состояний $\varphi_{m}$ неоднозначен, и путем диагонализации блока $\left\langle\varphi_{m}|\hat{H}| \varphi_{m^{\prime}}\right\rangle$ можно перейти от $\varphi_{m}$ к их линейным комбинациям $\Phi_{m}$, так что действительная матрица гамильтониана $H$ размерностью $n \times n$ представляется в следующем (каноническом) виде:

$$
H=\left(\begin{array}{cc}
0 & B^{t} \\
B & A
\end{array}\right)
$$

Здесь $A=\operatorname{diag}\left(A_{1}, A_{2}, \ldots, A_{n-1}\right)$ - диагональная матрица, $\quad A_{m-1}=\left\langle\Phi_{m}|\hat{H}| \Phi_{m}\right\rangle ; \quad B \quad$ - матрица размерности $(n-1) \times 1 \quad$ (вектор), $\quad B^{t}=\left(B_{1}, B_{2}, \ldots, B_{n-1}\right)$; $B_{m-1}=\left\langle\varphi_{1}\left|\hat{H}^{(1)}\right| \Phi_{m}\right\rangle$ - матричные элементы связи. Кроме того, энергия уровня $\varphi_{1}$ (с учетом возмущения) принята за начало отсчета, $H_{11}=\left\langle\varphi_{1}|\hat{H}| \varphi_{1}\right\rangle=0$.

Обратная задача может быть сформулирована следующим образом: основываясь на спектральных данных определить энергии „темных“ состояний $A_{m}$ и матричные элементы их связи со „светлым“ $B_{m}$. Число указанных величин $n-1+n-1=2 n-2$. Что касается данных эксперимента, то для $n$ линий конгломерата известно их положение $E_{k}^{\prime}$ и интенсивность $I_{k}^{\prime}$, всего $2 n$ величин. Однако, как следствие ортогональности матрицы $C$ сумма теоретических интенсивностей нормирована на $1, \sum_{k=1}^{n}\left(C_{1 k}\right)^{2}=1$. Этому отвечает равенство $\sum_{k=1}^{n} I_{k}=1$ для нормированных экспериментальных интенсивностей $I_{k}=I_{k}^{\prime} / \sum_{l=1}^{n} I_{l}^{\prime}$. Кроме того, энергия уровня $\varphi_{1}$ принята за начало отсчета, $H_{11}=0$, поэтому из $H=C E C^{t}$ следует равенство $\sum_{k=1}^{n} E_{k}\left(C_{1 k}\right)^{2}=0$. Этому соответствует то, что „центр тяжести“ наблюдаемого конгломерата $G=\sum_{k=1}^{n} E_{k}^{\prime} I_{k}$ должен отождествляться с положением уровня энергии состояния $\varphi_{1}$ относительно уровня основного состояния $\varphi_{0}$, и для наблюдаемых энергий $E_{k}$ относительно „центра тяжести“ $G\left(E_{k}=E_{k}^{\prime}-G\right)$ выполняется соотношение $\sum_{k=1}^{n} E_{k} I_{k}=0$. Таким образом, на экспериментальные величины $E_{k}^{\prime}$ и $I_{k}^{\prime}$ наложены два дополнительных условия, т.е. из наблюдаемых величин независимы только $2 n-2$. Отсюда следует, что обратная задача должна иметь однозначное решение.

Необходимо отметить, что обратная задача выглядит достаточно необычно и первоначально для ее решения применялся метод проб и ошибок [2,22] (с использованием данных для молекул нафталина и пиразина соответственно). Критерием подгонки параметров $A_{m}$ и $B_{m}$ служило совпадение интенсивностей рассчитанного и наблюдаемого спектров. В работах [23-28] для этих целей использовался метод функции Грина, в том числе для случая дискретных „темных“ состояний $[27,28]$.

Предварительная диагонализация матрицы „темных“ состояний ведет к каноническому виду (3) матрицы $H$, при этом однако возникает вопрос о интерпретации $A_{m}$ и $B_{m}[21,27,29,30]$. В рамках используемого линейного вариационного метода (1) величины $A_{m}$ - это энергии уровней „темных“ состояний $\Phi_{m}$, возмущенных за счет „взаимодействия“ между собой, а $B_{m}$ - матричные элементы связи этих состояний со „светлым“. Такого рода величины должны сопоставляться с независимыми экспериментальными или расчетными данными, например с энергиями обертонов и составных колебаний $[7,25]$. В рамках обратной задачи сведений о предварительной диагонализации „темных“ состояниях нет, поэтому мож- 
но пренебречь элементами $\left\langle\varphi_{m}\left|\hat{H}^{(1)}\right| \varphi_{m^{\prime}}\right\rangle$ при $m \neq m^{\prime}$, и тогда матрица $H$ будет иметь вид (3) и в исходном базисе „темных“ состояний $\varphi_{m}$. В аналогичном приближении

$$
\left\langle\varphi_{1}|\hat{H}| \varphi_{1}\right\rangle \approx\left\langle\varphi_{1}\left|\hat{H}^{(0)}\right| \varphi_{1}\right\rangle=0,
$$

т. е. начало отсчета - уровень невозмущенного „светлого“ состояния, а

$$
\begin{aligned}
A_{m-1}= & \left\langle\Phi_{m}|\hat{H}| \Phi_{m}\right\rangle-\left\langle\varphi_{1}|\hat{H}| \varphi_{1}\right\rangle \approx\left\langle\varphi_{m}\left|\hat{H}^{(0)}\right| \varphi_{m}\right\rangle+ \\
& +\left\langle\varphi_{m}\left|\hat{H}^{(1)}\right| \varphi_{m}\right\rangle-\left\langle\varphi_{1}\left|\hat{H}^{(1)}\right| \varphi_{1}\right\rangle \approx\left\langle\varphi_{m}\left|\hat{H}^{(0)}\right| \varphi_{m}\right\rangle
\end{aligned}
$$

трактуются как энергии невозмущенных „темных“ состояний, и соответственно $\quad B_{m-1}=\left\langle\varphi_{1}|\hat{H}| \Phi_{m}\right\rangle \approx$ $\approx\left\langle\varphi_{1}\left|\hat{H}^{(1)}\right| \varphi_{m}\right\rangle$ - как матричные элементы их связи со „светлым“.

Рассматриваемую задачу называют еще обратной диагонализацией, так как величины $A_{m}$ и $B_{m}$ будут известны, если найдено решение уравнения $H=C E C^{t}$, в котором диагональная матрица $E$ состоит из наблюдаемых энергий уровней, $E=\operatorname{diag}\left(\left\{E_{k}\right\}\right)$, а элементы первой строки матрицы $C$ удовлетворяют условию $\left(C_{1 k}\right)^{2}=I_{k}$. В нашей работе [31] найден не требующий итерационных процедур алгебраический метод решения рассматриваемой обратной задачи. Существенным образом он опирается на решение уравнения на собственные значения для матрицы $H$ при $A_{m}=$ const и построение на этой основе ортогональной матрицы $X$, у которой квадрат элементов ее первой строки равен интенсивности наблюдаемых линий, $\left(C_{1 k}\right)^{2}=I_{k}$. В настоящей работе будет показано, что такого рода ортогональная матрица $X$ может быть найдена без использования диагонализации промежуточных матриц, как это необходимо делать в [31], а только с помощью плоских вращений Якоби.

\section{Простой резонанс Ферми, $n=2$}

Для простого резонанса Ферми [32] или его вибронного аналога при $n=2$ матрица $H$ имеет вид (3), но „темное“ состояние только одно, предварительной диагонализации не требуется, и $A$ и $B-$ числа. В прямой задаче матрица $H$ приводится к диагональному виду преобразованием подобия с

$$
C=\left(\begin{array}{cc}
c & s \\
-s & c
\end{array}\right)
$$

где $c=\cos \theta, s=\sin \theta, \theta=(1 / 2) \operatorname{arctg}(2 B / A)$. Соответствующие интенсивности переходов $\varphi_{0} \rightarrow \Psi_{1}, \varphi_{0} \rightarrow \Psi_{2}$ даются как $I_{1}=c^{2}$ и $I_{2}=s^{2}$. С другой стороны, в обратной задаче матрица $H$ определяется с помощью преобразования подобия $H=C E C^{t}, E=\operatorname{diag}\left(E_{1}, E_{2}\right)$, и теперь для $C$ естественно определить косинусы и синусы как $c=\sqrt{I_{1}}$ и $s=\sqrt{I_{2}}$. Величины $A$ и $B$ тогда даются из наблюдаемых энергий и интенсивностей соотношениями $A=\left(E_{2}-E_{1}\right)\left(I_{1}-I_{2}\right), B=\left(E_{2}-E_{1}\right) \sqrt{I_{1} I_{2}}$.

\section{Ортогональное преобразование на основе вращений Якоби. Первый этап алгоритма}

Кроме функций $\Phi_{k}$ вида (1) мы можем рассматривать функции

$$
\Theta_{k}=\sum_{l=1}^{n} X_{l k} \varphi_{l},
$$

так что матрица $X$, составленная из коэффициентов $X_{l k}$, ортогональная. Таких наборов функций $\Theta_{k}$, конечно, бесконечное количество. Отличие функций $\Theta_{k}$ от функций $\Psi_{k}$ состоит в том, что в общем случае матрица гамильтониана $\hat{H}$ на функциях $\Theta_{k},\left\langle\Theta_{k}|\hat{H}| \Theta_{k^{\prime}}\right\rangle$, не имеет диагонального вида, и поэтому ее элементы не могут быть непосредственно соотнесены с наблюдаемыми энергиями. Это верно уже в силу того, что наблюдается $n$ переходов с энергиями $E_{k}$, которых при $n>2$ недостаточно для задания $n(n+1) / 2$ элементов симметричной матрицы $\left\langle\Theta_{k}|\hat{H}| \Theta_{k^{\prime}}\right\rangle$. Наложим теперь на функции (4) требование $X_{1 k}=C_{1 k}$, т.е. пусть первая строка матриц $X$ и $C$ совпадает. Тогда функции $\Theta_{k}$ и $\Psi_{k}$ различаются линейными комбинациями функций „темных“ состояний. Кроме того, с учетом предварительной диагонализации вместо функций „темных“ состояний $\varphi_{m}$ в (4) могут стоять функции $\Phi_{m}$ :

$$
\Theta_{k}=X_{1 k} \varphi_{1}+\sum_{m=2}^{n} X_{m k} \Phi_{m} .
$$

Так как „светлым“ является только состояние $\varphi_{1}$, то переходы $\varphi_{0} \rightarrow \Theta_{k}$ имеют такую же интенсивность, что и переходы $\varphi_{0} \rightarrow \Psi_{k}$. Это обстоятельство и будет использоваться нами для нахождения алгоритма решения поставленной обратной задачи.

Рассмотрим матрицу $X_{1}$ в виде элементарного плоского вращения Якоби:

$$
X_{1}=\left(\begin{array}{rrrrr}
c_{1} & s_{1} & 0 & \ldots & 0 \\
-s_{1} & c_{1} & 0 & \ldots & 0 \\
0 & 0 & 1 & \ldots & 0 \\
. & . & . & . & . \\
0 & 0 & 0 & \ldots & 1
\end{array}\right)
$$

Этому преобразованию соответствуют преобразованные функции $\Theta_{1}$ и $\Omega_{2}$ :

$$
\begin{aligned}
& \Theta_{1}=c_{1} \varphi_{1}-s_{1} \Phi_{2}, \\
& \Omega_{2}=s_{1} \varphi_{1}+c_{1} \Phi_{2} .
\end{aligned}
$$

Определим косинусы и синусы $c_{1}$ и $s_{1}$ как

$$
c_{1}=\sqrt{I_{2}^{(1)}}, \quad s_{1}=\sqrt{1-I_{1}^{(1)}} .
$$

Здесь для согласования с нижеприведенными формулами обозначения для интенсивностей изменены: $I_{k}^{(1)} \equiv I_{k}$. 
Из (6), (7) следует, что, во-первых, переход $\varphi_{0} \rightarrow \Theta_{1}$ имеет такую же интенсивность, что и переход $\varphi_{0} \rightarrow \Psi_{1}$, и поэтому в дальнейшем достаточно искать преобразования, которые оставляют неизменной функцию $\Theta_{1}$, но затрагивают только функции $\Omega_{2}, \Phi_{3}, \ldots, \Phi_{n}$. Вовторых, видно, что оставшаяся часть интенсивности, $1-I_{1}^{(1)}$, сосредоточена в переходе $\varphi_{0} \rightarrow \Omega_{2}$, и его следует считать источником заимствования в остальные переходы. Таким образом, теперь нужно искать перераспределение интенсивности от этого перехода в переходы в состояния $\Theta_{2}, \Theta_{3}, \ldots, \Theta_{n}$, так чтобы их относительная интенсивность совпадала с относительной интенсивностью переходов в состояния $\Psi_{2}, \Psi_{3}, \ldots, \Psi_{n}$. Тем самым рассматриваемая задача о заимствовании интенсивности от одного перехода $\varphi_{0} \rightarrow \varphi_{1}$ в $n$ переходов свелась к задаче о заимствовании интенсивности от одного перехода $\varphi_{0} \rightarrow \Omega_{2}$ в $n-1$ переход.

Определим новые относительные интенсивности $I_{k}^{(2)}=I_{k}^{(1)} /\left(1-I_{k}^{(1)}\right)$ для $k=2,3, \ldots, n$ с выполнением нормировки $\sum_{k=2}^{n} I_{k}^{(2)}=1$.

Рассмотрим матрицу преобразования $X_{2}$, аналогичную (5),

$$
X_{2}=\left(\begin{array}{cccccc}
1 & 0 & 0 & 0 & \ldots & 0 \\
0 & c_{2} & s_{2} & 0 & \ldots & 0 \\
0 & -s_{2} & c_{2} & 0 & \ldots & 0 \\
0 & 0 & 0 & 1 & \ldots & 0 \\
. & . & . & . & . & \\
0 & 0 & 0 & 0 & \ldots & 1
\end{array}\right)
$$

и определим $c_{2}$ и $s_{2}$ следующим образом:

$$
c_{2}=\sqrt{I_{2}^{(2)}}, \quad s_{2}=\sqrt{1-I_{2}^{(2)}} .
$$

Матрице (8) соответствуют преобразованные функции

$$
\begin{aligned}
& \Theta_{2}^{\prime}=c_{2} \Omega_{2}-s_{2} \Phi_{3}, \\
& \Omega_{3}^{\prime}=s_{2} \Omega_{2}+c_{2} \Phi_{3} .
\end{aligned}
$$

В свою очередь произведению матриц $X_{1} X_{2}$ отвечают функции

$$
\begin{aligned}
& \Theta_{2}=c_{2}\left(s_{1} \varphi_{1}+c_{1} \Phi_{2}\right)-s_{2} \Phi_{3}, \\
& \Omega_{3}=s_{2}\left(s_{1} \varphi_{1}+c_{1} \Phi_{2}\right)+c_{2} \Phi_{3} .
\end{aligned}
$$

Интенсивность перехода $\varphi_{0} \rightarrow \Theta_{2}$ определяется величиной $c_{2} s_{1}$, которая равна

$$
\begin{aligned}
c_{2} s_{1} & =\sqrt{I_{2}^{(2)}} \sqrt{1-I_{1}^{(1)}}=\left(\sqrt{I_{2}^{(1)}} / \sqrt{1-I_{1}^{(1)}}\right) \sqrt{1-I_{1}^{(1)}}= \\
& =\sqrt{I_{2}^{(1)}}=\sqrt{I_{2}} .
\end{aligned}
$$

Таким образом, преобразование $X_{1} X_{2}$ дает две функции $\Theta_{1}$ и $\Theta_{2}$, так что интенсивности $I_{1}$ и $I_{2}$ переходов $\varphi_{0} \rightarrow \Theta_{1}$ и $\varphi_{0} \rightarrow \Theta_{2}$ совпадают с интенсивностями переходов $\varphi_{0} \rightarrow \Psi_{1} \quad$ и $\varphi_{0} \rightarrow \Psi_{2}$. Функция $\Omega_{3}$ имеет компоненту, пропорциональную $\varphi_{1}$ :

$$
\begin{aligned}
s_{2} s_{1}= & \sqrt{1-I_{2}^{(2)}} \sqrt{1-I_{1}^{(1)}}=\sqrt{1-I_{2}^{(1)} /\left(1-I_{1}^{(1)}\right)} \times \\
& \times \sqrt{1-I_{1}^{(1)}}=\sqrt{1-I_{1}^{(1)}-I_{2}^{(1)}}=\sqrt{1-I_{1}-I_{2}} .
\end{aligned}
$$

и тем самым переход $\varphi_{0} \rightarrow \Omega_{3}$ можно считать источником дальнейшего перераспределения интенсивности теперь уже в переходы в $n-2$ состояния $\Theta_{3}, \Theta_{4}, \ldots, \Theta_{n}$.

Точно также могут быть построены матрицы $X_{3}, X_{4}, \ldots, X_{n-1}$, аналогичные матрицам (5), (8), а их произведение даст матрицу $X=X_{1} X_{2} \ldots X_{n-1}$. Матрице преобразования $X$ соответствуют функции $\Theta_{k}$, и интенсивность переходов $\varphi_{0} \rightarrow \Theta_{k}$ такая же, как и интенсивность переходов $\varphi_{0} \rightarrow \Psi_{k}$.

\section{Второй этап алгоритма}

После того как матрица $X$, элементы первой строки которой удовлетворяют условиям $\left(X_{1 k}\right)^{2}=I_{k}$ и $\sum_{k=1}^{n}\left(X_{1 k}\right)^{2}=1$, построена, умножим равенство $C^{t} H C=E$ слева на $X$ и справа на $X^{t}$. Результат будет иметь следующий блочный вид:

$$
X C^{t} H C X^{t}=X E X^{t}=\left(\begin{array}{cc}
0 & D^{t} \\
D & F
\end{array}\right) .
$$

В формуле (9) $F-$ матрица размерности $(n-1)(n-1) ; \quad D-$ матрица размерности $(n-1) \times 1$; $D^{t}=\left(D_{1}, D_{2}, \ldots, D_{n-1}\right) .\left(X E X^{t}\right)_{11}=0$ в силу того, что $\sum_{k=1}^{n} E_{k}\left(X_{1 k}\right)^{2}=\sum_{k=1}^{n} E_{k} I_{k}=0$.

По способу построения преобразования $X$ матрица $F$ - симметричная, действительная. Сформулируем для нее задачу на собственные значения:

$$
F Z=Z A
$$

где $A=\operatorname{diag}\left(A_{1}, A_{2}, \ldots, A_{n-1}\right)-$ диагональная матрица. Тогда $F=Z A Z^{t}, Z^{t}=Z^{-1}$.

Умножая выражение (9) слева на $Y$ и справа на $Y^{t}$, где $Y=\left(\begin{array}{cc}1 & 0 \\ 0 & Z^{t}\end{array}\right)$, получим

$$
Y X C^{t} H C X^{t} Y^{t}=Y X E X^{t} Y^{t}=\left(\begin{array}{cc}
0 & \left(Z^{t} D\right)^{t} \\
Z^{t} D & A
\end{array}\right),
$$

т. е. найдены как искомое преобразование $C$, так и матрица $H$ гамильтониана, имеющая требуемую структуру (3):

$$
\begin{gathered}
C=Y X, \\
H=(Y X) E(Y X)^{t} .
\end{gathered}
$$

Первая строка матрицы $C$ совпадает с первой строкой матрицы $X, C_{1 k}=X_{1 k}$, и, следовательно, требование для 

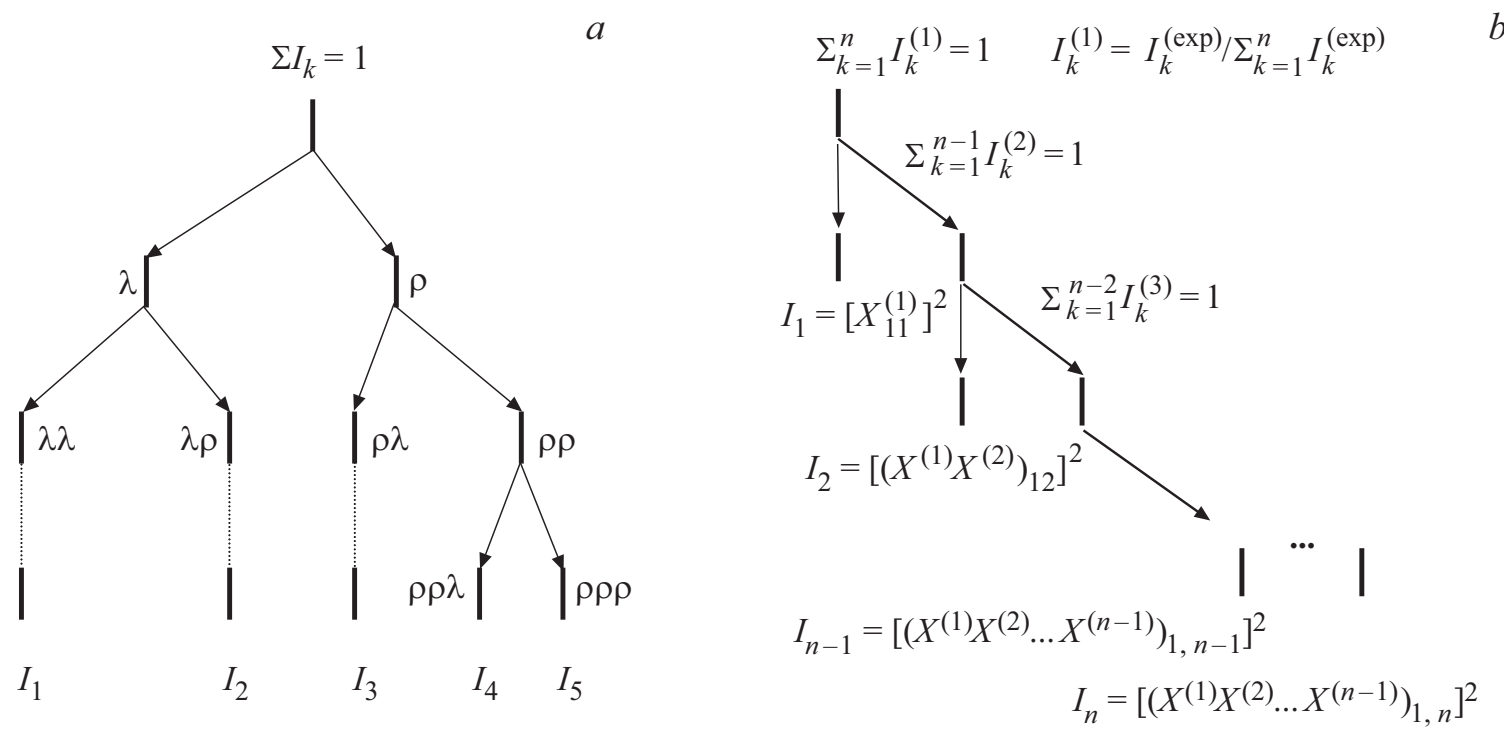

Перераспределение интенсивностей по алгоритму [31] (для $n=5)(a)$ и алгоритму настоящей статьи $(b)$.

интенсивностей выполнено: $\left(C_{1 k}\right)^{2}=\left(X_{1 k}\right)^{2}=I_{k}$. Собственные значения $A_{m}$ задачи $(10)$ дают энергии возмущенных (невозмущенных в вышеобсужденном приближении) „темных“ состояний, а соответствующие матричные элементы их связи со „светлым“ состоянием вычисляются из уравнения $B=Z^{t} D$ или

$$
B_{m}=\sum_{j=1}^{n-1} D_{j} Z_{j m} .
$$

\section{Связь с алгоритмом [31]. Однозначность определения параметров $A_{m}$ и $B_{m}$}

В нашей работе [31] было найдено, что характеристический полином $P_{n}(e)=\operatorname{det}\{H-e I\}$ матрицы $H$ канонического вида (3) при $A_{m}=a$ имеет следующие корни:

$$
\begin{gathered}
e_{1}=a / 2-\left[(a / 2)^{2}+b^{2}\right]^{1 / 2}, \quad e_{2}=\ldots e_{n-1}=a, \\
e_{n}=a / 2+\left[(a / 2)^{2}+b^{2}\right]^{1 / 2},
\end{gathered}
$$

где $b^{2}=\sum_{m=1}^{n-1} B_{m}^{2}$. Первая строка матрицы собственных векторов, соответствующая этим корням, содержит только два ненулевых значения, $\quad\left(e_{n} /\left(e_{n}^{2}+b^{2}\right)^{1 / 2}, 0, \ldots, 0, e_{1} /\left(e_{1}^{2}+b^{2}\right)^{1 / 2}\right), \quad$ и $\left(C_{11}\right)^{2}+\left(C_{1 n}\right)^{2}=1$. Из этой особенности следует, что интенсивность от исходного перехода в „светлое“ состояние первоначально заимствуется только в два перехода, но далее можно считать, что каждый из них в свою очередь становится источником заимствования в еще два перехода и т.д. до исчерпывания за некоторое число шагов. В [31] показано, каким образом на каждом
Таблица 1. Относительные энергии $E_{k}$ и нормированные интенсивности $I_{k}(k=1,2, \ldots, 12)$ конгломерата линий в области 0-0-перехода $S_{0} \rightarrow S_{1}$ молекулы пиразина [18]

\begin{tabular}{c|c|c|c|c|c}
\hline$k$ & $E_{k}, 10^{-2} \mathrm{~cm}^{-1}$ & $I_{k}$ & $k$ & $E_{k}, 10^{-2} \mathrm{~cm}^{-1}$ & $I_{k}$ \\
\hline 1 & -9.52 & 0.012 & 7 & -0.76 & 0.248 \\
2 & -8.39 & 0.007 & 8 & -0.19 & 0.042 \\
3 & -4.80 & 0.040 & 9 & 0.17 & 0.129 \\
4 & -1.79 & 0.042 & 10 & 2.03 & 0.017 \\
5 & -1.22 & 0.122 & 11 & 2.46 & 0.248 \\
6 & -1.08 & 0.037 & 12 & 2.77 & 0.055
\end{tabular}

шаге (применительно к исходной задаче) вычислять параметр $a$ и набор $B_{m}$ из комбинаций наблюдаемых величин $\left\{E_{k}\right\}$ и $\left\{I_{k}\right\}$. Процедура включает в себя также диагонализацию промежуточных матриц. В итоге получена ортогональная матрица $X$, у которой квадрат элементов ее первой строки равен интенсивности наблюдаемых линий, $\left(X_{1 k}\right)^{2}=I_{k}$, как и матрица $X E X^{t}$ вида (9). Такой способ аналогичен классическому подходу в алгебраической проблеме собственных значений [33], при котором сначала находятся собственные значения матрицы, а затем ее собственные вектора. В отличие от этого в настоящей статье представлен алгоритм, где с самого начала ищется преобразование подобия $X$ с условием $\left(X_{1 k}\right)^{2}=I_{k}$, для чего используются вращения Якоби. Рисунок иллюстрирует последовательное перераспределение интенсивности, соответствующее построению матриц $X$ с помощью обоих алгоритмов.

Очевидно, что матрицы $X$ на основе двух алгоритмов различаются между собой. Из приведенного рисунка ясна также принципиальная возможность построения 
Таблица 2. Энергии „темных“ колебательных состояний триплетного состояния $A_{m}$ и матричные элементы их вибронной и спинорбитальной связи $B_{m}(m=1,2, \ldots, 11)$ с электронным состоянием $S_{1}$ молекулы пиразина $\left(\right.$ все в $\left.10^{-2} \mathrm{~cm}^{-1}\right)$

\begin{tabular}{|c|c|c|c|c|c|c|c|}
\hline \multicolumn{3}{|c|}{$A_{m}$} & \multicolumn{3}{|c|}{$B_{m}$} & $F_{m m}$ & $D_{m}$ \\
\hline Настоящая работа & {$[27]$} & {$[22]$} & Настоящая работа & {$[27]$} & {$[22]$} & \multicolumn{2}{|c|}{ Настоящая работа } \\
\hline-9.408 & -9.411 & -9.400 & 0.953 & 0.965 & 0.944 & -9.398 & 1.049 \\
\hline-8.321 & -8.323 & -8.306 & 0.737 & 0.762 & 0.787 & -8.323 & 0.714 \\
\hline-4.597 & -4.605 & -4.587 & 0.959 & 0.957 & 0.957 & -4.582 & 1.015 \\
\hline-1.724 & -1.730 & -1.718 & 0.279 & 0.280 & 0.287 & -1.682 & 0.456 \\
\hline-1.130 & -1.137 & -1.121 & -0.179 & 0.179 & 0.180 & -0.946 & 0.640 \\
\hline-1.008 & -1.014 & -1.001 & 0.253 & 0.253 & 0.257 & -0.982 & 0.361 \\
\hline-0.296 & -0.303 & -0.287 & -0.432 & 0.432 & 0.437 & 0.056 & 0.979 \\
\hline-0.023 & -0.029 & -0.010 & 0.425 & 0.425 & 0.420 & -0.011 & 0.395 \\
\hline 1.464 & 1.467 & 1.471 & 1.411 & 1.410 & 1.408 & 0.843 & 0.704 \\
\hline 2.073 & 2.067 & 2.088 & 0.261 & 0.264 & 0.277 & 2.062 & 0.062 \\
\hline 2.729 & 2.723 & 2.745 & 0.188 & 0.188 & 0.187 & 2.720 & 0.066 \\
\hline
\end{tabular}

Примечание. Экспериментально значения $E_{k}$ определяются с точностью до двух знаков после десяточной точки, последний знак для величин $A_{m}$ и $B_{m}$ следует считать лишним. Он приведен только для сравнения с данными расчетов [22] и [27].

некоторой ортогональной матрицы $X$ на основе комбинации двух алгоритмов (практически, однако в этом нет никакой необходимости). В соответствии с этим набор матриц $X E X^{t}$ вида (9) может быть получен достаточно большим количеством способов, и каждая пара из них связана преобразованием подобия. Искомый вид матрицы $H$ (3) получается после диагонализации блока $F$ в (9), а в целом - после ряда преобразований подобия над диагональной матрицей $E$. Можно утверждать, что параметры $\left\{A_{m}\right\}$ и $\left\{B_{m}\right\}$ через параметры $\left\{E_{k}\right\}$ и $\left\{I_{k}\right\}$ выражаются однозначно, число и тех, и других одинаково, $2 n-2$.

В целом, решение обратной задачи для сложного вибронного аналога резонанса Ферми как в работе [31], так и в настоящей работе опирается на матричные методы, характерные для алгебраической проблемы собственных значений, оно альтернативно методике на основе функции Грина $[27,28]$.

\section{Расчеты матричных элементов $A_{m}$ и $B_{m}$ для молекул порфиринов и пиразина}

Найденный алгоритм реализован нами в виде фортрановской программы. Она намного проще, чем программа на основе алгоритма [31]. Можно сделать следующее замечание. В программе для проверки правильности работы, после того как найдена матрица $H$ из уравнения $H=C E C^{t}, C=Y X$, предусмотрена диагонализация $H \bar{C}=\bar{C} \bar{E}$ и проверка условий $\left|\bar{E}_{k}-E_{k}\right|<\varepsilon_{1}$, $\left|\left(\bar{C} C^{t}-I_{k l}\right)\right|<\varepsilon_{2}$ где $\varepsilon_{1}$ и $\varepsilon_{2}$ задаются машинной точностью. В расчетах с использованием метода функции Грина $[27,28]$ первоначально итерационным образом вычисляются величины $A_{m}$, а затем $\left(B_{m}\right)^{2}$ (дальнейший выбор знака $\pm \sqrt{B_{m}^{2}}$ несуществен, что связано с изменением знака базисных функций), и также производится решение уравнения $H \bar{C}=\bar{C} \bar{E}$ [27], но в критерии
$\left|\bar{E}_{k}-E_{k}\right|<\varepsilon_{1}, \quad \varepsilon_{1}$ зависит от точности итерационной процедуры.

С помощью фортрановской программы проведены расчеты параметров вибронной связи $A_{m}$ и $B_{m}$ на основе данных по положению и интенсивности линий в спектрах возбуждения флуоресценции в области 0-0-перехода $S_{0} \rightarrow S_{2}$ трех порфириновых соединений: мезо-тетраазапорфина, мезо-тетрапропилпорфина [12] и $2,3,12,13-$-тетраметилдибензо $[g, q]$ порфина [13] (для двух типов внедрения в поликристаллическую матрицу каждой молекулы). Полученные результаты по $A_{m}$ (энергиям возмущенных колебательных состояний электронного уровня $S_{1}$ ) и по $B_{m}$ (соответствующим матричным элементам связи с электронным состоянием $S_{2}$ ) полностью совпали с данными, ранее вычисленными на основе алгоритма [31] (см. табл. 2, 3 в [12] и табл. 2 в [13]). Тем самым подтвержден ряд следующих выводов, актуальных для понимания вибронного взаимодействия.

(1). Интервал между „чисто“ электронными уровнями $S_{2}$ и $S_{1}$ (характерные значения $2200-3000 \mathrm{~cm}^{-1}$ ), чувствительный к микроокружению (изменения в пределах $\left.100-200 \mathrm{~cm}^{-1}\right)$, играет роль параметра модуляции для резонирующих электронно-колебательных состояний в диапазоне $\sim 1000 \mathrm{~cm}^{-1}$.

(2). Хотя различия между системами наблюдаемых линий спектра возбуждения флуоресценции для двух главных типов внедрения молекул в матрицу весьма значительны, энергии большинства их составных колебательных состояний различаются на характерную величину $\sim 10 \mathrm{~cm}^{-1}$.

(3). Типичная величина матричных элементов вибронной связи у рассмотренных молекул порфиринов составляет $20-30 \mathrm{~cm}^{-1}$ во всем диапазоне резонанса. Это значение сопоставимо с величиной, полученной для молекулы нафталина $\left(10-20 \mathrm{~cm}^{-1}[23]\right)$, в то время как, согласно [2], они в 5-10 раз больше. 
В табл. 1 приведены исходные экспериментальные данные для расчета вибронной и спин-орбитальной связи электронного состояния $S_{1}$ с колебательно возбужденными триплетными состояниями молекулы пиразина [18]. Необходимо отметить, что эти данные, представленные количественно наборами $\left\{E_{k}\right\}$ и $\left\{I_{k}\right\}$, послужили значительным толчком [27] к развитию методики на основе формализма функции Грина.

В табл. 2 наши результаты сравнены с данными расчетов на основе методов проб и ошибок [22] и функции Грина [27]. Как видно, значения $A_{m}$ и $B_{m}$ с точностью до двух знаков после десятичной точки совпадают во всех методах. Как и в [19], отметим, что для матричных элементов связи $B_{m}$ имеет место значительный разброс.

Проведенные расчеты выполнены для нумерации переходов как в табл. 1. Поэтому можно отметить также, что диагональные элементы $F_{m m}$ матрицы $F(9)$ близки к величинам $A_{m}$, особенно для „крыльев“ конгломерата, достаточно отдаленных от его центра тяжести при 0. Этого нельзя сказать о величинах $D_{m}$ и $B_{m}$, особенно вблизи центра конгломерата, где запутанность состояний велика, и второй этап алгоритма, связанный с диагонализацией матрицы $F$, играет существенную роль.

Развитую методику можно было бы применить и к другим сложным спектрам [3-11,14-17,20], к сожалению, многие экспериментальные данные не представлены в численном виде.

\section{Заключение}

Таким образом, в настоящей работе на основе матричных методов представлено точное решение обратной задачи, которая предусматривает определение из данных для наблюдаемого конгломерата линий, возникающего за счет сложного резонанса типа резонанса Ферми (разрешен только один переход в „светлое“ состояние), энергий уровней „темных“ состояний и матричных элементов их связи со „светлым“ состоянием. Алгоритм решения существенным образом использует плоские вращения Якоби для построения ортогональной матрицы, у которой квадрат элементов ее первой строки равен интенсивности наблюдаемых линий.

\section{Список литературы}

[1] Герцберг Г. Электронные спектры и строение многоатомных молекул. М.: Мир, 1969. С. 68-71.

[2] Wessel J., McClure D.S. // Mol. Cryst. Liq. Cryst. 1980. V. 58. P. 121.

[3] Narva D.L., McClure D.S. // Chem. Phys. 1981. V. 56. P. 167.

[4] Ruth A.A., Doherty W.G., Brint R.P. // Chem. Phys. Lett. 2002. V. 352. P. 191.

[5] Ohta N., Baba H., Marconi G. // Chem. Phys. Lett. 1987. V. 133. P. 222.

[6] Rouillé G., Krasnokutski S., Huisken F., Hennig T., Sukhorukov O., Staicu A. // J. Chem. Phys. 2004. V. 120. P. 6028.
[7] Tan X., Salama F. // J. Chem. Phys. 2005. V. 123. P. 014312.

[8] Rouillé G., Arold M., Staicu A., Krasnokutski S., Huisken F., Henning T., Tan X., Salama F. // J. Chem. Phys. 2007. V. 126. P. 174311.

[9] Deperasińska I., Zehnacker A., Lahmani F., Borowicz P., Sepiot J. // J. Phys. Chem. A. 2007. V. 111. P. 4252.

[10] Поведайло В.А., Яковлев Д.Л. // ЖПС. 2008. Т. 75. С. 319.

[11] Singh A., Huang W.-Y., Scheiner P., Johnson L.W. // J. Phys. Chem. A. 2006. V. 110. P. 6454.

[12] Арабей С.М., Кузьмицкий В.А., Соловьев К.Н. // Опт. и спектр. 2007. Т. 102. С. 765.

[13] Arabei S.M., Kuzmitsky V.A., Solovyov K.N. // Chem. Phys. 2008. V. 352. P. 197.

[14] Шкирман С.Ф., Соловьев К.Н. // ЖПС. 2008. Т. 75. С. 796.

[15] Koyanagi M., Nakashima K., Goodman L. // Chem. Phys. 1985. V. 92. P. 435.

[16] Winkler I.C., Hanson D.M. // Chem. Phys. 1984. V. 91. P. 173.

[17] Nakashima K., Koyanagi M. // Bull. Chem. Soc. Japan. 1985. V. 58. P. 2000.

[18] Van der Meer B.J., Jonkman H.T., Kommandeur J., Meerts W.L., Majewski W.A. // Chem. Phys. Lett. 1982. V. 92. P. 565.

[19] Kommandeur J., Majewski W.A., Meerts W.L., Pratt D.W. // Ann. Rev. Phys. Chem. 1987. V. 38. P. 433.

[20] Alvarez-Valtierra L., Tan X.-Q., Pratt D.W. // J. Phys. Chem. A. 2007. V. 111. P. 12802.

[21] Макаров А.А., Малиновский А.Л., Рябов Е.А. // УФН. 2012. T. 182. C. 1047.

[22] Van der Meer B.J., Jonkman H.T., Kommandeur J. // Laser Chem. 1983. V. 2. P. 77.

[23] Langhoff C.A., Robinson G.W. // Chem. Phys. 1974. V. 6. P. 34.

[24] Hong H.-K. // Chem. Phys. 1975. V. 9. P. 1.

[25] Berg J.O. // Chem. Phys. Lett. 1976. V. 41. P. 547.

[26] Ziv A.R., Rhodes W. // J. Chem. Phys. 1976. V. 65. P. 4895.

[27] Lawrence W.D., Knight A.E.W. // J. Phys. Chem. 1985. V. 89. P. 917.

[28] Lehman K.K. // J. Phys. Chem. 1991. V. 95. P. 7556.

[29] Lawrence W.D., Knight A.E.W. // J. Phys. Chem. 1991. V. 95. P. 7557.

[30] Bittenger K.L., Field R.W. // J. Chem. Phys. 2010. V. 132. P. 134302.

[31] Кузьмицкий В.А. // Опт. и спектр. 2006. Т. 101. С. 711.

[32] Лисииа М.П., Яремко А.М. Резонанс Ферми. Киев: Наукова думка, 1984.

[33] Уилкинсон Джс.Х. Алгебраическая проблема собственных значений. М.: Наука, 1970. 